\title{
Improved Bounds on the Crossing Number of Butterfly Network
}

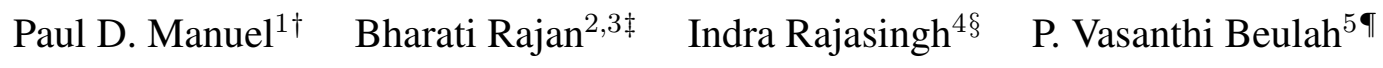 \\ ${ }^{1}$ Department of Information Science, Kuwait University, Safat 13060, Kuwait \\ ${ }^{2}$ Department of Mathematics, Loyola College, Chennai - 600 034, India \\ ${ }^{3}$ School of Electrical Engineering and Computer Science, The University of New Castle, NSW 2308, Australia \\ ${ }^{4}$ School of Advanced Sciences, VIT University, Chennai - 600 127, India \\ ${ }^{5}$ Department of Mathematics, Queen Mary's College, Chennai - 600 004, India \\ received $28^{\text {th }}$ January 2011, revised $15^{\text {th }}$ March 2013, accepted $12^{\text {th }}$ April 2013.
}

We draw the $r$ - dimensional butterfly network with $\frac{1}{4} 4^{r}+O\left(r 2^{r}\right)$ crossings which improves the previous estimate given by Cimikowski (1996). We also give a lower bound which matches the upper bound obtained in this paper.

Keywords: drawing of a graph, crossing number, butterfly network

\section{Introduction}

The crossing number $\operatorname{cr}(G)$ of a graph $G$ is the minimum number of crossings of its edges among all drawings of $G$ in the plane. It is an important measure of the non-planarity of a graph [12], with applications in discrete and computational geometry [11]. The crossing number of a graph corresponding to the VLSI circuit has strong influence on the area layout as well as on the number of wire contact cuts that should be minimized. Discoveries of Leighton in the early 1980's made this, an area of high importance in the theoretical computer science [1, 6]. The chip area required for the realization of an electrical network is closely related to the crossing number of the underlying graph. In information visualization, one of the most important measures of the quality of a graph drawing is the number of crossings [9]. The problem is NP-hard [5].

Cimikowski [4] has given an upper bound for the crossing number of the butterfly network as

$$
\frac{3}{2} 4^{r}+O\left(r 2^{r}\right)
$$

\footnotetext{
†Email: pauldmanuel @gmail . com. Supported by UNESCO-HP under "Brain Gain Initiative" Scheme (Project Number is 3250039600) and Kuwait University (XX06/09).

‡Email: bharatirajan@gmail.com.

§Email: indrarajasingh@yahoo.com

IEmail: vbeulah@yahoo.com. Supported by the Minor Research Project - No.F.MRP-3712/11(MRP/UGC-SERO), P.No. 345, University Grants Commission, India.
}

1365-8050 (c) 2013 Discrete Mathematics and Theoretical Computer Science (DMTCS), Nancy, France 
where $r$ denotes the dimension of the network. In 2008, Manuel et al. [7] announced a drawing $(D)$ of this butterfly network for which we obtained [10] an improved bound as

$$
\operatorname{cr}\left(B F_{r}\right) \leq 4^{r}+O\left(r 2^{r}\right), r \geq 3 .
$$

In this paper we propose a new drawing $(\phi)$ of the butterfly network in the plane with

$$
\frac{1}{4} 4^{r}+O\left(r 2^{r}\right)
$$

number of crossings. We also give a lower bound for the crossing number of the butterfly network.

\section{Butterfly Network}

The set $V$ of vertices of an $r$-dimensional butterfly correspond to pairs $[w, i]$, where $i, 0 \leq i \leq r$ is the dimension or level of the vertex and $w$ is an $r$-bit binary number that denotes the row of the vertex. Two vertices $[w, i]$ and $\left[w^{\prime}, i^{\prime}\right]$ are linked by an edge if and only if $i^{\prime}=i+1$ and either:

1. $w$ and $w^{\prime}$ are identical, or

2. $w$ and $w^{\prime}$ differ in precisely the $i^{\text {th }}$ bit.

The $r$-dimensional butterfly network denoted by $B F_{r}$ has $(r+1) 2^{r}$ vertices and $r 2^{r+1}$ edges. It has $r+1$ levels and there are $2^{r}$ vertices in each level. Each vertex on level 0 and level $r$ is of degree 2 . All the other vertices are of degree 4 .

\subsection{Proposed New Drawing}

The new drawing $\phi$ of the butterfly network is constructed from the one $(D)$ obtained by Manuel et al. [7] using some drawing pattern.

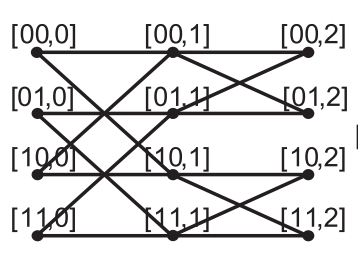

(a)

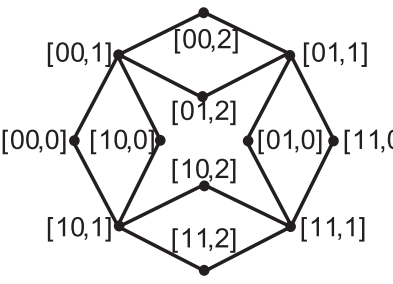

(b)

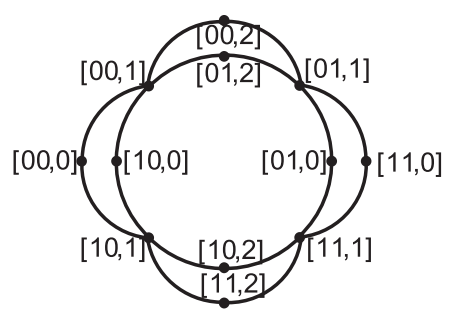

(c)

Fig. 1: (a) Original drawing of $B F_{2}$ (b) Drawing $D$ proposed by Manuel et al. (c) Drawing $\phi$ which we propose in this paper.

It is easily seen from Figure $1(b)$ and $(c)$ that the $D\left(B F_{2}\right)$ can be drawn as $\phi\left(B F_{2}\right)$. We describe next the procedure for the drawing of $\phi\left(B F_{3}\right)$. Consider imaginary lines through the level 0 and level 2 vertices of $\phi\left(B F_{2}\right)$. We call these lines as 0-line (horizontal line) and 2-line (vertical line) respectively. 
The 0-line divides the drawing of $\phi\left(B F_{2}\right)$ into two symmetrical parts say the upper half $\mathrm{UH}$ and the lower half LH. See Figure $2(a)$. Reflect the lower half about the 0-line so that no two vertices overlap and the level 2 vertices of LH continue to lie on the 2 -line. See Figure $2(b)$. Call this diagram as $\phi^{\prime}\left(B F_{2}\right)$. Take a copy of $\phi^{\prime}\left(B F_{2}\right)$, call it $\phi^{\prime \prime}\left(B F_{2}\right)$ and flip it vertically and place it below $\phi^{\prime}\left(B F_{2}\right)$. Now join the level 0 vertices of $\phi^{\prime}\left(B F_{2}\right)$ with the corresponding vertices of $\phi^{\prime \prime}\left(B F_{2}\right)$ by means of 4-cycles which we call as binding 4-cycles. The resulting diagram is called $\phi\left(B F_{3}\right)$ and is shown in Figure $2(c)$. The binding 4 -cycles are shown by means of broken lines.

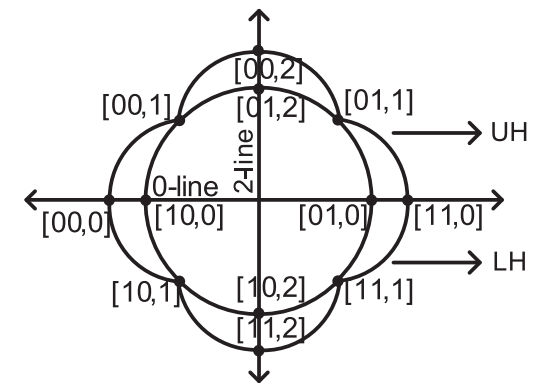

(a)

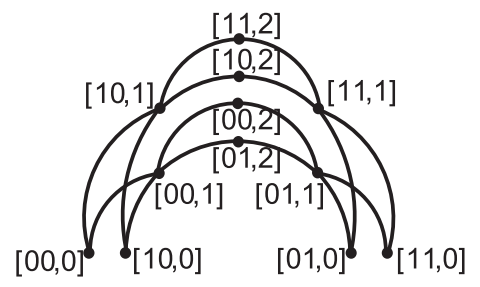

(b)

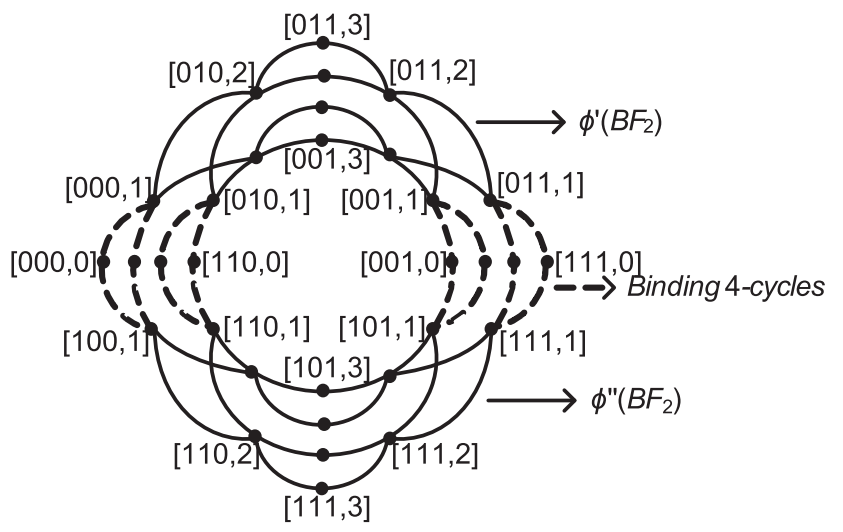

(c)

Fig. 2: (a) $\phi\left(B F_{2}\right)$ (b) $\phi^{\prime}\left(B F_{2}\right)$ (c) $\phi\left(B F_{3}\right)$

The vertices of this graph are labeled as follows: Vertices of $\phi^{\prime}\left(B F_{2}\right)$ and $\phi^{\prime \prime}\left(B F_{2}\right)$ are of the form $\left[u_{1} u_{2}, r\right]$, where $u_{1}, u_{2} \in\{0,1\}$ and $0 \leq r \leq 2$. Rename each vertex $\left[u_{1} u_{2}, r\right]$ of $\phi^{\prime}\left(B F_{2}\right)$ as $\left[0 u_{1} u_{2}, r+\right.$ 1] and each of $\phi^{\prime \prime}\left(B F_{2}\right)$ as $\left[1 u_{1} u_{2}, r+1\right]$. The new vertices in the binding 4-cycles are of the form $\left[u_{1} u_{2} u_{3}, 0\right]$ where $u_{1}, u_{2}, u_{3} \in\{0,1\}$. The procedure is recursive. 
We have the following observations with respect to the drawing $\phi\left(B F_{r}\right)$.

1. In a $\phi\left(B F_{r}\right)$ level 0 vertices are placed horizontally and level $r$ vertices are placed vertically. The graph is symmetric about level 0 vertices as well as the level $r$ vertices.

2. $\phi\left(B F_{2}\right)$ is planar. But $\phi^{\prime}\left(B F_{2}\right)$ is non planar with 2 crossings.

3. $\phi^{\prime}\left(B F_{r}\right)$ is symmetric about the level $r$ vertices.

4. $\phi\left(B F_{r}\right)$ contains $\phi^{\prime}\left(B F_{r-1}\right), \phi^{\prime \prime}\left(B F_{r-1}\right)$ and $2^{r-1}$ binding 4-cycles. These binding 4-cycles do not contribute to crossing number.

5. The edges incident on the level 0 vertices of $\phi\left(B F_{r}\right)$ which lie in its lower half contribute additionally to the crossing number of $\phi^{\prime}\left(B F_{r}\right)$. The number of such edges is $2^{r}$.

Note: In what follows $D$ will denote the drawing of $B F_{r}$ introduced by Manuel et al. [7] and $\phi$ will denote a drawing of $B F_{r}$ drawn according to the procedure mentioned above. Similarly $\phi^{\prime}$ and $\phi^{\prime \prime}$ denote their reflected copies as shown in Figure $2(b)$.

\subsection{An Improved Upper Bound}

Cimikowski [4] has obtained an upper bound for the crossing number of $B F_{r}$. Following his techniques, based on a combinatorial analysis of the structure of the underlying graph, we obtain an upper bound for the crossing number of $\phi\left(B F_{r}\right)$.

Theorem 1 [4 Let $B F_{r}$ be an r-dimensional butterfly network. Then,

$$
\operatorname{cr}\left(B F_{r}\right) \leq \frac{3}{2} 4^{r}-r 2^{r}-3.2^{r}+1 .
$$

Theorem 2 [10] Let D be a drawing of an r-dimensional butterfly network. Then

$$
c r_{D}\left(B F_{r}\right)=4^{r}-r 2^{r+1}, r \geq 3 .
$$

For the drawing $\phi$ of an $r$-dimensional butterfly network, we have the following lemma.

Lemma 1 Let $\phi\left(B F_{r}\right)$ and $\phi^{\prime}\left(B F_{r}\right)$ be the drawings of $r$-dimensional butterfly network. Then

$$
c r_{\phi^{\prime}}\left(B F_{r}\right)=\operatorname{cr}_{\phi}\left(B F_{r}\right)+2^{r-1}\left(2^{r-1}-1\right) .
$$

Proof: The edges incident on the level 0 vertices of $\phi\left(B F_{r}\right)$ and lying in its lower half contribute additionally to the crossing number of $\phi^{\prime}\left(B F_{r}\right)$. The number of such edges is $2^{r}$. Therefore, ${ } r_{\phi^{\prime}}\left(B F_{r}^{\prime}\right)=$ $c r_{\phi}\left(B F_{r}\right)+m$, where $m$ denotes the number of additional crossings while forming $\phi^{\prime}\left(B F_{r}\right)$. Moreover $\phi^{\prime}\left(B F_{r}\right)$ is symmetric about the level $r$ vertices. Hence without loss of generality, we consider the edges ( $2^{r-1}$ edges) to the left of the level $r$ vertices of $\phi^{\prime}\left(B F_{r}\right)$ and proceed to count the number of crossings contributed by these edges. First edge contributes 0 to the number of crossings, second edge 1 , third edge 2 and so on. Therefore $2^{r-1}$ edges contribute to the number of crossings as follows: $0,1,2,3 \ldots\left(2^{r-1}-1\right)$. Hence $m=2\left[0+1+2+3+\ldots+\left(2^{r-1}-1\right)\right]=2^{r-1}\left(2^{r-1}-1\right)$. 
In Figure 3, the small square boxes represent the additional crossings in $\phi^{\prime}\left(B F_{3}\right)$.

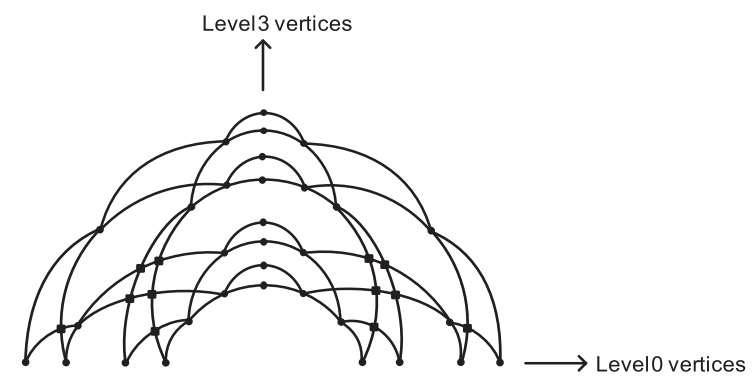

Fig. 3: Additional crossings in the formation of $\phi^{\prime}\left(B F_{3}\right)$

Theorem 3 For the drawing $\phi$ of an r-dimensional butterfly network,

$$
c r_{\phi}\left(B F_{r}\right)=\frac{1}{4} 4^{r}-r 2^{r-1}, r \geq 3 .
$$

Proof: We prove the theorem by induction on $r$. It is clear from the drawing $\phi\left(B F_{3}\right)$ that the number of crossings is $4=\frac{1}{4} 4^{3}-3 \cdot 2^{3-1}$. Assume that the result is true for dimension $r=k, 3<k<r$. Let $\phi\left(B F_{k}\right)$ be a drawing of a $k$-dimensional butterfly network. By symmetry and by Lemma 1 it is clear that

$$
\begin{aligned}
\operatorname{cr}_{\phi}\left(B F_{k}\right) & =2 c r_{\phi^{\prime}}\left(B F_{k-1}\right) \\
& =2\left[c r_{\phi}\left(B F_{k-1}\right)+2^{k-2}\left(2^{k-2}-1\right)\right] \\
& =2\left[\left\{\frac{1}{4} 4^{k-1}-(k-1) 2^{k-2}\right\}+2^{k-2}\left(2^{k-2}-1\right)\right] \\
& =\frac{1}{4} 4^{k}-k 2^{k-1} .
\end{aligned}
$$

Theorems 1, 2 and 3 give the crossing numbers corresponding to three different drawings of $r$-dimensional butterfly network. We observe that $\phi\left(B F_{r}\right)$ gives the least value among these three and hence we have the following result.

Theorem 4 Let $G$ be an r-dimensional butterfly network. Then,

$$
\operatorname{cr}(G) \leq \frac{1}{4} 4^{r}-r 2^{r-1}, r \geq 3 .
$$




\subsection{Lower Bound}

We first solve the crossing number problem for $B F_{3}$. We begin with the following results.

Theorem 5 ([3], Euler's formula) In a connected plane graph $G$ with $n$ vertices, $\varepsilon$ edges and $f$ faces (regions), $n-\varepsilon+f=2$.

Theorem 6 ([3]) If $G$ is a connected plane graph with girth $g$, then $g f \leq 2 \varepsilon$.

Lemma 2 Let $G$ be a 3-dimensional butterfly network. Then $\operatorname{cr}(G) \geq 4$.

Proof: Let $t$ be the smallest non-negative integer such that the removal of some $t$ edges from the graph $G$ results in a planar graph $G_{t}$. The graph $G_{t}$ is a connected spanning subgraph of the graph of $G$ with 32 vertices and $64-t$ edges. By Theorem $5 n-\varepsilon+f=2$. Here $n=32$ and $\varepsilon=64-t$. Hence $G_{t}$ has $34-t$ regions. Also by Theorem 6 , since $G_{t}$ has girth four, $4(34-t) \leq 2(64-r)$. Hence $t \geq 4$ and therefore $\operatorname{cr}(G) \geq 4$.

Lemma 2 and Theorem 4 imply the following result.

Theorem $7 \quad \operatorname{cr}\left(B F_{3}\right)=4$.

We next proceed to obtain a lower bound for the crossing number which is fairly close to the upper bound obtained in Theorem 4 .

The following terminologies related to butterfly network and some of the graph parameters are required in order to determine the lower bound for the crossing number of $B F_{r}$ for large values of $r$.

In a butterfly network the vertices on level 0 are called the inputs of the network, and the vertices on level $r$ are called the outputs. An $n$ - input butterfly network has $n$ vertices in level 0 . We say $B F_{r}$ is an $2^{r}$ - input butterfly network as there are $2^{r}$ vertices in level 0 .

The bisection width of a graph $G$ denoted by $b w(G)$, is the minimum number of edges whose removal divides $G$ into two parts having at most $2|V| / 3$ vertices each.

The best upper bound we obtained in this paper is of order $O\left(4^{r}\right)$. A lower bound of matching order can be simply obtained by combining the known facts.

Theorem 8 Let $G$ be an $r$-dimensional butterfly network. Then

$$
\operatorname{cr}(G) \geq \frac{1}{59} 4^{r}-r 2^{r}+2^{r-1}
$$

It is known [8] that for an $n$-vertex graph with the degree sequence $\left(d_{1}, d_{2} \ldots d_{n}\right)$ and bisection width $b w(G)$,

$$
c r(G) \geq \frac{1}{40} b w^{2}(G)-\frac{1}{16} \sum d_{v}^{2}
$$


It is also shown in [2] that for an $2^{r}$-input butterfly network, the bisection width is given by,

$$
b w(G)=0.82 \times 2^{r}
$$

upto error terms. Combining the results (1) and (2), we get immediately that,

$$
\begin{aligned}
c r(G) & \geq \frac{1}{40} b w^{2}(G)-\frac{1}{16} \sum d_{v}^{2} \\
& =\frac{1}{40}\left(0.82 \times 2^{r}\right)^{2}-\frac{1}{16}\left\{(r-1) 2^{r} \cdot 16+2 \cdot 2^{r} \cdot 4\right\} \\
& =\frac{1}{59} 4^{r}-r 2^{r}+2^{r-1} .
\end{aligned}
$$

\section{Conclusion}

Theorem 1 provides a $\frac{3}{2} 4^{r}+O\left(r 2^{r}\right)$ bound for the crossing number of an $r$-dimensional butterfly network. The drawing proposed in this paper drastically reduces the upper bound to $\frac{1}{4} 4^{r}+O\left(r 2^{r}\right)$. We have also obtained a matching lower bound as $\frac{1}{59} 4^{r}+O\left(r 2^{r}\right)$. It would be interesting to explore such tight bounds for other interconnection networks too.

\section{Acknowledgements}

We thank the referees for their insightful comments and valuable suggestions on the improvement of this paper.

\section{References}

[1] S. N. Bhatt, F. T. Leighton, A Framework for Solving VLSI Graph Layout Problems, J. Comput. System Sci, 28, 300-343, 1984.

[2] C. F. Bornstein, A. Litman, B. M. Maggs, R. K. Sitaraman, T. Yatzkar, On the Bisection Width and Expansion of Butterfly Networks, Theory of Computing Systems 34, 494-518, 2001.

[3] J. A. Bondy, U. S. R. Murty, Graph Theory with Applications, University of Waterloo, Canada, 2008.

[4] R. Cimikowski, Topological Properties of Some Interconnection Network Graphs, Congr. Numer., 121, 19-32, 1996.

[5] M. R. Garey, D. S. Johnson. Crossing Number is NP-Complete, SIAM J. Alg. Disc. Meth, 4, 312$316,1983$.

[6] F. T. Leighton, Complexity Issues in VLSI, MIT Press, Cambridge, Mass., 1983.

[7] P. D. Manuel, I. Mostafa, I. Rajasingh, B. Rajan, An Efficient Representation of Benes Network and its Applications, Journal of Discrete Algorithms, 6, 11-19, 2008.

[8] J. Pach, F. Shahrokhi, M. Szegedy, Applications of the Crossing Number, Algorithmica, 16, 111-117, 1996. 
[9] H. C. Purchase, R. F. Cohen, and M. I. James. An experimental study of the basis for graph drawing algorithms. ACM Journal of Experimental Algorithmics, 2(4), 1997. http://www.jea.acm.org/1997/PurchaseDrawing.

[10] B. Rajan, I. Rajasingh, P. Manuel, P. V. Beulah, On the Crossing Number of Butterfly Networks, Proceedings of the International Conference on Mathematics and Computer Science, India, 587$580,2010$.

[11] L. A. Székely, Crossing numbers and hard Erdôs problems in discrete geometry. Combin.Probab. Comput., 6(3), 353-358, 1997.

[12] L. A. Székely, A Successful Concept for Measuring Nonplanarity of Graphs: the Crossing Number, Discrete Math. 276, 331-352, 2004. 